PAPER

\title{
Neurophysiological predictors of long term response to AChE inhibitors in AD patients
}

\author{
V Di Lazzaro, A Oliviero, F Pilato, E Saturno, M Dileone, C Marra, S Ghirlanda, F Ranieri, \\ G Gainotti, P Tonali
}

J Neurol Neurosurg Psychiatry 2005;76:1064-1069. doi: 10.1136/jnnp.2004.051334

See end of article for authors' affiliations ........................

Correspondence to: Vincenzo Di Lazzaro, Istituto di Neurologia, Universitò Cattolica, L.go A. Gemelli 8, 00168 Rome, Italy; vdilazzaro@ rm.unicatt.it

Received 5 August 2004 Revised version received 26 November 2004 Accepted

26 November 2004
Background: In vivo evaluation of cholinergic circuits of the human brain has recently been introduced using a transcranial magnetic stimulation (TMS) protocol based on coupling peripheral nerve stimulation with motor cortex TMS (short latency afferent inhibition, SAl). SAl is reduced in Alzheimer's disease (AD) and drugs enhancing cholinergic transmission increase SAl.

Methods: We evaluated whether SAl testing, together with SAI test-retest, after a single dose of the acetylcholinesterase (AChE) inhibitor rivastigmine, might be useful in predicting the response after 1 year treatment with rivastigmine in $16 \mathrm{AD}$ patients.

Results: Fourteen AD patients had pathologically reduced SAI. SAl was increased after administration of a single oral dose of rivastigmine in $A D$ patients with abnormal baseline $S A I$, but individual responses to rivastigmine varied widely, with SAl change ranging from an increase in inhibition of $\sim 50 \%$ of test size to no change. Baseline SAl and the increase in SAl after a single dose of rivastigmine were correlated with response to long term treatment. A normal SAI in baseline conditions, or an abnormal SAI in baseline conditions that was not greatly increased by a single oral dose of rivastigmine, were invariably associated with poor response to long term treatment, while an abnormal SAI in baseline conditions in conjunction with a large increase in SAl after a single dose of rivastigmine was associated with good response to long term treatment in most of the patients.

Conclusions: Evaluation of SAl may be useful for identifying AD patients likely to respond to treatment with AChE inhibitors.
$\mathrm{T}$ he hypothesis of significant cholinergic dysfunction in Alzheimer's disease $(\mathrm{AD})$ has provided a rationale for pharmacological treatments based on drugs that may enhance cholinergic neurotransmission, such as acetylcholinesterase (AChE) inhibitors. Although the benefit of AChE inhibitors is rather limited, these drugs are at the front line of treatment for patients with $\mathrm{AD} .^{1}$ A clinically significant response to AChE inhibitors is observed in only $30-60 \%$ of $\mathrm{AD}$ patients, depending on the compound. ${ }^{2}$ To date, no laboratory test is available which can identify patients who are likely to respond to these drugs.

In vivo demonstrations of a cholinergic deficit in $\mathrm{AD}$ patients have been provided recently by our group ${ }^{3}$ using a technique that may give direct information about the function of some cholinergic circuits in the human cerebral cortex..$^{5}$ This technique relies on the phenomenon of short latency afferent inhibition (SAI) of the motor cortex. ${ }^{6}$ SAI is normal or even slightly enhanced in different neurological disorders such as Parkinson's disease. ${ }^{78}$

In our previous studies we found that rivastigmine, a centrally acting drug that blocks AChE, may increase SAI in $\mathrm{AD}$ patients towards normal values. ${ }^{34}$ Moreover, preliminary data on a small group of six $\mathrm{AD}$ patients suggest that the assessment of SAI before and after the administration of a single oral dose of rivastigmine might be a useful tool for predicting the clinical response after 1 year of treatment. ${ }^{4}$

In $\mathrm{AD}$ patients there is also a tendency to have a less pronounced short latency cortico-cortical inhibition, a different form of inhibition produced by paired cortical magnetic stimulation. $^{3}{ }^{4}$ Cortico-cortical inhibition is believed to involve GABAA activity. ${ }^{10}$ The slight abnormality of cortico-cortical inhibition in $\mathrm{AD}$ patients is not modified by the administration of rivastigmine. ${ }^{3}$
In the present study we evaluate for a larger group of $\mathrm{AD}$ patients whether SAI testing together with SAI test-retest, performed after a single oral dose of rivastigmine, might be useful in order to predict the response to long term treatment. The possibility of predicting the response to treatment would have a significant impact on the management of $\mathrm{AD}$ patients by improving the use of healthcare resources and preventing the exposure of potential nonresponder $\mathrm{AD}$ patients to the risk of $\mathrm{AChE}$ inhibitor related side effects.

\section{METHODS}

\section{Patients}

In this prospective study, we initially recruited 20 patients with a diagnosis of probable $\mathrm{AD}$ according to the NINCDSADRDA criteria. ${ }^{11}$ The criteria for inclusion were (a) absence of other major medical illnesses and (b) symptom onset no longer than 5 years before the study. None of these patients was included in our previous study. ${ }^{4}$

All the AD patients selected were able to understand and carry out the simple task required for this electrophysiological study, that is to remain fully relaxed. All the patients were right handed as assessed with the Edinburgh inventory. ${ }^{12}$ None of the patients had been treated with drugs that may have modulated cerebral cortex excitability in the 30 days before participating in this electrophysiological study and none of the patients had been treated with cholinesterase

Abbreviations: AChE, acetylcholinesterase; AD, Alzheimer's disease; FDI, first dorsal interosseous muscle; GDS, Global Deterioration Scale; ISI, interstimulus interval; MEP, motor evoked potential; RAVLT, Rey's Auditory Verbal Learning Test; SAl, short latency afferent inhibition; TMS, transcranial magnetic stimulation 
inhibitors before participating in the study, which was performed according to the Declaration of Helsinki and approved by the ethics committee of the Medical Faculty of the Catholic University in Rome. Patients and their caregivers gave their informed consent before participation.

The main clinical and demographic characteristics of the $\mathrm{AD}$ patients are set out in table 1. Electrophysiological findings obtained in patients were compared with those obtained in 12 neurologically healthy age matched control subjects. The mean (SD) age of the patients was 70.5 (6.9) years, while that of controls was 73.1 (5.4) years.

\section{SAl by somatosensory input from the hand}

Magnetic stimulation was performed using two high power Magstim 200 magnetic stimulators (Magstim, Whitland, Dyfed, UK) connected to the BiStim module (Magstim) throughout all measurements. A figure-of-eight coil with external loop diameters of $9 \mathrm{~cm}$, was held over the right motor cortex at the optimum scalp position to elicit motor responses in the contralateral first dorsal interosseous muscle (FDI). The induced current flowed in a postero-anterior direction. The optimal position was marked on the scalp to ensure identical placement of the coil throughout the experiment. Motor evoked potentials (MEPs) were recorded through two $9 \mathrm{~mm}$ diameter $\mathrm{Ag}$ - $\mathrm{AgCl}$ electrodes with the active electrode over the motor point of the muscle and the reference on the metacarpophalangeal joint of the index finger. MEPs were amplified and filtered (bandwidth $3 \mathrm{~Hz}-$ $3 \mathrm{kHz}$ ) by D360 amplifiers (Digitimer, Welwyn Garden City, Herts, UK). Data were collected on a computer with a sampling rate of $10 \mathrm{kHz}$ per channel and stored for later analysis using a CED 1401 A-D converter (Cambridge Electronic Design, Cambridge, UK).

SAI was studied using the technique we have recently described. ${ }^{6}$ Conditioning peripheral stimuli were single pulses $(200 \mu \mathrm{s})$ of electrical stimulation applied through bipolar electrodes to the median nerve at the wrist (cathode proximal). The intensity of the conditioning peripheral stimulus was set at just over the motor threshold for evoking a visible twitch of the thenar muscles. The intensity of the test cortical magnetic shock was adjusted to evoke an MEP in a relaxed FDI with an amplitude of approximately $1 \mathrm{mV}$ peak-to-peak.

The conditioning stimulus to the peripheral nerve preceded the magnetic test stimulus. The afferent inhibition induced by the peripheral conditioning stimulus was tested using the following pulse configuration: magnetic stimulus alone, and

Table 1 Demographic characteristics and neuropsychological test scores

\begin{tabular}{lll}
\hline & AD & Cut off scores \\
\hline Age & $70.5(6.9)$ & \\
Educational level & $7.9(2.9)$ & \\
Time from onset disease (months) & $26.8(16.4)$ & \\
Gender (male/female) & $8 / 12$ & \\
MMSEE & $19.1(5.5)$ & $>24$ \\
RAVLT, immediate recall & $22.7(8.3)$ & $>28.56$ \\
RAVLT, delayed recall & $2.5(3)$ & $>4.64$ \\
RAVLT, recognition (hits) & $10.6(4.2)$ & $>12$ \\
RAVLT, recognition (false alarms) & $11.3(9.1)$ & $<2$ \\
Immediate visual memory & $13.6(4.9)$ & $>14.95$ \\
Raven's coloured matrices & $14.4(5.9)$ & $>18.98$ \\
Constructive praxis & $6.6(3.6)$ & $>8$ \\
Constructive praxis (with & $53.4(13.9)$ & $>67$ \\
landmarks) & & \\
Phonological verbal fluency & $14.3(11.2)$ & $>21.5$ \\
\hline
\end{tabular}

The results shown are the neuropsychological test mean scores and standard deviations obtained by AD patients and cut off scores in a normal population matched for age and educational level. MMSE, Mini Mental State Examination; RAVLT, Rey's Auditory Verbal Learning Test. peripheral conditioning stimulus preceding the cortical magnetic stimulus at different interstimulus intervals (ISIs). ISIs were determined relative to the latency of the N20 component of the somatosensory evoked potential obtained after stimulation of the left median nerve. The active electrode for recording the N20 potential was attached $3 \mathrm{~cm}$ behind C4 (10-20 system) and the reference was $3 \mathrm{~cm}$ behind C3. A total of 500 responses were averaged to identify the latency of the N20 peak. ISIs from the latency of the N20 plus $2 \mathrm{~ms}$ to the latency of the $\mathrm{N} 20$ plus $8 \mathrm{~ms}$ were investigated in steps of $1 \mathrm{~ms}$. Each recording consisted of 40 trials. Magnetic stimulation of the motor cortex was performed on every trial; in 35 of the trials selected at random cortical magnetic stimulation was preceded at one of the seven investigated ISIs by a conditioning stimulus to the median nerve at the wrist. We calculated an average (based on five trials each) of the MEP obtained after cortical magnetic stimulation alone and of the MEP obtained by conditioning cortical magnetic stimulus with a peripheral stimulus to the median nerve at the wrist at the seven different ISIs studied. The subject was given audio-visual feedback at high gain to assist in maintaining complete relaxation. The amplitude of the conditioned MEP was expressed as a percentage of the amplitude of the test MEP. We averaged the percentage of inhibition of the conditioned responses at the seven different ISIs to obtain a grand mean of SAI. This was done because we have previously demonstrated that the abnormality of SAI in AD is evident at all these ISIs, but the grand mean has the advantage of reducing variability. ${ }^{3}$ The test with seven ISIs can be completed in a few minutes.

\section{Test-retest variability of SAI}

To evaluate the test-retest variability of SAI, in five control subjects (mean (SD) age: 29.6 (5.4) years) we performed a baseline SAI study and repeated it at an interval of $3 \mathrm{~h}$.

\section{Effects of AChE inhibition on SAI}

At the time of patient enrolment we repeated the measurement of SAI after the administration of a single dose (3 $\mathrm{mg}$ ) of rivastigmine, an AChE inhibitor commonly used for treatment of $\mathrm{AD}$, in all patients with abnormal SAI. SAI was measured before and $2.4 \mathrm{~h}$ after the administration, when AChE inhibition in the CSF is maximal. ${ }^{13}$ All patients were started on chronic treatment with rivastigmine (6$9 \mathrm{mg} /$ die) immediately after the electrophysiological tests.

\section{Neuropsychological examination}

All patients enrolled in the study underwent the MMSE (Mini Mental State Examination) and an extensive neuropsychological test battery, including tests of episodic verbal memory (Rey's Auditory Verbal Learning Test, RAVLT), ${ }^{14}$ immediate visual memory, constructional praxis, verbal fluency, abstract reasoning (Raven's Progressive Matrices '47), ${ }^{15}$ and a test of executive function sensitive to frontal lobe damage (temporal rule induction). ${ }^{16}$

In RAVLT we evaluated the immediate recall score, the delayed recall score and two scores of forced delayed recognition: (a) the number of correctly recognised target words (hits); and (b) the number of incorrectly recognised distractor words (false alarms).

After l year of treatment, the same neuropsychological assessment was repeated in all patients.

\section{Follow up}

The outcome of the treatment was assessed using the Global Deterioration Scale (GDS) ${ }^{17}$ and the values at the beginning of the treatment were compared with the values obtained 
after 1 year of treatment. A stable or a decreased score was considered the marker of a response to the treatment.

We also evaluated the neuropsychological response to the treatment as the number of tests on which score was improved or unchanged after l year of treatment.

\section{Statistical analysis}

We used Mann-Whitney tests to compare SAI in AD patients and the control group, and Wilcoxon's matched pair test to compare SAI in $\mathrm{AD}$ patients before and after administration of a single oral dose of rivastigmine. A value of SAI within 2 SD from the mean of controls was considered normal. An increase in SAI after rivastigmine above 2 SD from the mean change observed in controls in the test-retest variability study, was considered beyond the intrinsic variability of this test. We used the permutation test (which makes no assumptions about the statistical distribution of variables ${ }^{18}$ ) to compare the clinical response (change in GDS score) to the treatment in patients with normal and abnormal baseline SAI.

In patients with abnormal baseline SAI who underwent SAI test-retest after a single oral dose of rivastigmine, we used Kendall's rank test to evaluate correlations between neuropsychological response to treatment and each of the following variables: change in SAI after a single dose of rivastigmine, MMSE at enrolment, age of subject, age at $\mathrm{AD}$ diagnosis, and time since $\mathrm{AD}$ diagnosis. The same test was used to evaluate the correlation between the clinical and neuropsychological response measures, and the correlation between change in score in each of 10 neuropsychological tests and variation in SAI after a single dose of rivastigmine, in patients with abnormal baseline SAI. In the latter case, to correct for multiple tests we adopted a significance level of $\mathrm{p}<0.0051$. All other tests employ a significance level of $\mathrm{p}<0.05$. All tests are two tailed.

\section{RESULTS}

MEPs in control subjects were inhibited when the median nerve stimulus was given before the cortical stimulus at an interval corresponding to the N20 latency plus $2 \mathrm{~ms}$ to N20 latency plus $8 \mathrm{~ms}$ (fig 1 ). The mean amount of inhibition over this period was larger in controls (responses (SD) reduced to $45.3 \%(16.2 \%)$ of test size) than in $\mathrm{AD}$ patients $(86.2(21.3 \%)$; $\mathrm{p}<0.001$, two tailed Mann-Whitney test) (fig 1). Examination of individual data (table 2 ) shows that 14 patients had results outside the normal range (that is, above $77.7 \%$ ), while six patients fell within the normal range.

\section{Test-retest variability of SAI in normal subjects}

To evaluate the test-retest variability of SAI, in five control subjects we performed a baseline SAI study and repeated it, without rivastigmine administration, at an interval of $3 \mathrm{~h}$. The mean (SD) amplitude of the conditioned response was $42.4 \%(16.7 \%)$ of test size at the baseline study and $42.1 \%$ $(18.8 \%)$ at an interval of $3 \mathrm{~h}(\mathrm{p}>0.05$, two tailed Wilcoxon matched pairs test). The change in the amplitude of the conditioned response ranged from $1.7 \%$ to $5.8 \%$ of test size with a mean (SD) change in the amplitude of the conditioned response of $4.6 \%(1.6 \%)$ of test size.

Therefore, a change greater than $8 \%$ of test size (mean plus 2 SD in control subjects) in the amplitude of the conditioned response after rivastigmine in $\mathrm{AD}$ patient was considered beyond the intrinsic variability of this test.

\section{Effects of AChE inhibition in AD patients}

SAI was increased after the administration of a single oral dose of rivastigmine in $\mathrm{AD}$ patients with abnormal baseline SAI. The mean (SD) amplitude of the conditioned response was $95.5 \%(17.2 \%)$ of control size before rivastigmine and
$74.9 \%(14.6 \%)$ after rivastigmine $(\mathrm{p}<0.001$, two tailed Wilcoxon matched pairs test). Individual responses to rivastigmine varied widely, with change in SAI ranging from an increase in inhibition of about $50 \%$ of test size to no change (table 2). Twelve of the 14 patients with abnormal SAI had an increase in size of $\geqslant 8 \%$ of test size.

\section{Follow up}

After a few weeks, four of the patients initially recruited (nos. $4,5,6$, and 13 in table 2) stopped taking rivastigmine due to adverse side effects. Thus the fully evaluable population consisted of 16 patients (three patients with normal SAI and 13 patients with abnormal SAI). Only the data of these patients were further analysed.

A positive clinical response, defined as decreased or unchanged score on the GDS, was observed in eight patients $(50 \%)$. All these patients had an abnormal SAI and all of them had an increase in SAI after a single dose of rivastigmine of $\geqslant 8 \%$ of test size. The remaining eight patients showed progression of disease. Three of these patients had a normal baseline SAI, two had an abnormal SAI but presented a small change in SAI after rivastigmine, and three patients had an abnormal SAI and an increase in SAI after a single dose of rivastigmine of $\geqslant 8 \%$ of test size. Overall, the clinical response was significantly better in patients with abnormal baseline SAI ( $p<0.05$, two tailed permutation test).

After 1 year of treatment with rivastigmine, the $13 \mathrm{AD}$ patients with abnormal SAI at enrolment improved or remained stable on an average of 6.1 neuropsychological tests out of 10. The three AD patients with normal SAI, in contrast, improved or remained stable on an average of only 2.3 tests.

Considering all patients, neuropsychological and clinical measures of treatment outcome were significantly correlated (Kendall's $\mathrm{t}=-0.48, \mathrm{p}=0.0 \mathrm{l}$, two tailed test; considering only patients with abnormal baseline SAI: Kendall's $\mathrm{t}=-0.77, \mathrm{p}<0.0005$, two tailed test).

Change in SAI was positively correlated with an improvement in all 10 neuropsychological tests performed (all Kendall's rank correlations positive: $\mathrm{p}<10^{-8}$, two tailed binomial test), although only three correlations were statistically significant after correcting for multiple tests (immediate and delayed recall of RAVLT and phonological verbal fluency; table 3). Among patients with abnormal baseline SAI, change in SAI after a single oral dose of rivastigmine was strongly correlated with the number of neuropsychological tests on which score was improved or unchanged after 1 year of treatment (fig 2; Kendall's t $=0.92$, $\mathrm{p}<0.001$, two tailed test). The following variables did not significantly correlate with the clinical measure of treatment outcome: subject age, subject age at $\mathrm{AD}$ diagnosis, time since AD diagnosis, MMSE at enrolment ( $p$ between 0.06 and 0.85 , two tailed Kendall's rank tests).

\section{DISCUSSION}

In our previous studies we have found that SAI is reduced in $\mathrm{AD}$ patients and that it can be, at least in part, restored by administration of AChE inhibitors. ${ }^{3}$ Here we ask whether the evaluation of SAI and the evaluation of the acute effects of a single oral dose of rivastigmine might be useful to predict the response to long term treatment with this drug in $\mathrm{AD}$ patients.

Most of our patients ( $\sim 70 \%)$ had an abnormal baseline SAI. SAI is a test sensitive to the excitability of some cholinergic circuits in the human motor cortex and its abnormality is probably correlated with the most consistently demonstrated deficit in AD that involves reduced cholinergic activity. ${ }^{3}$ Therefore, the number of patients with abnormal SAI conceivably reflects the percentage of patients with a 


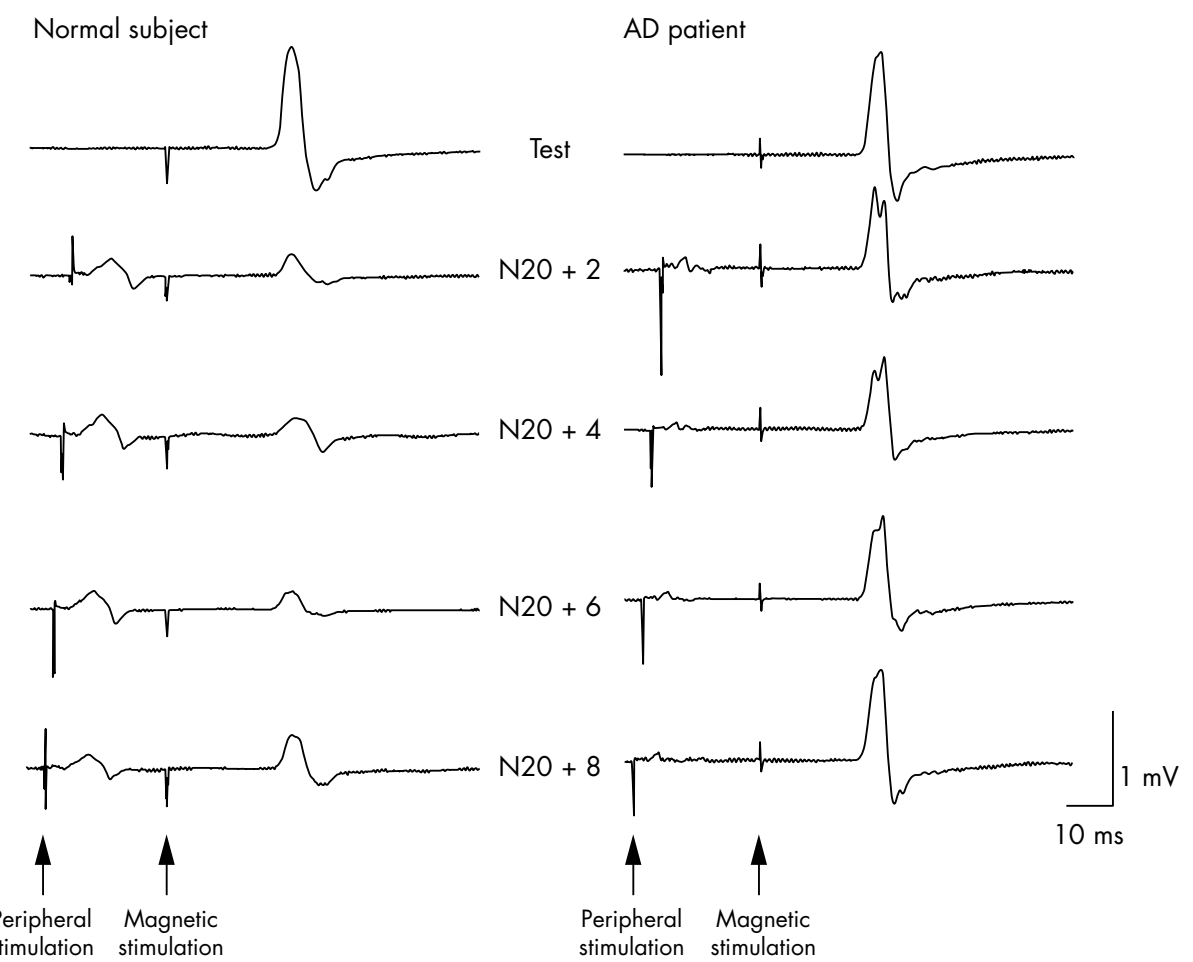

Figure 1 SAl produced by median nerve stimulation in one control subject (left) and in one patient with $A D$ (right). The top traces show the average (of five trials each) of MEP responses evoked in the FDI by cortical magnetic stimulation alone, lower traces show the average (of five trials each) of MEP responses evoked by cortical magnetic stimulation conditioned by a median nerve stimulus with an ISI corresponding to the N20 latency plus 2, 4, 6, and $8 \mathrm{~ms}$. In the control subject, the median nerve conditioning stimulus suppresses the MEP response evoked by cortical magnetic stimulation. In the AD patient, median nerve stimulation produces no clear inhibition.

significant cholinergic dysfunction among the patients with a clinical diagnosis of AD. However, it is still unknown whether different neurotransmitters such as glutamate, GABA, or dopamine are also involved in the regulation of SAI.

In the patients with abnormal SAI, afferent inhibition could be increased within hours of the administration of rivastigmine. However, the change in SAI after the administration of rivastigmine varied widely between individual patients. We considered the change in SAI in $\mathrm{AD}$ patients to be beyond the intrinsic variability when it was greater than 2 SD above the mean change observed in control subjects. In our previous study, we also tested the effect of a single oral dose of rivastigmine in control subjects. ${ }^{4}$ We observed only a very slight increase in SAI after rivastigmine, probably due to a "floor" effect, because the baseline levels of inhibition are strong in healthy subjects. ${ }^{4}$ However, it should be noted that the test-retest reliability of SAI and the effects of rivastigmine were evaluated in control subjects younger than the patients

\begin{tabular}{|c|c|c|c|c|}
\hline Patient & $\begin{array}{l}\text { Baseline SAI (\% of } \\
\text { control MEP) }\end{array}$ & $\begin{array}{l}\text { Increase in SAI after } \\
\text { rivastigmine (\% of } \\
\text { control MEP) }\end{array}$ & $\begin{array}{l}\text { Score on the GDS } \\
\text { (baseline/follow up) }\end{array}$ & $\begin{array}{l}\text { Number of neuropsychological } \\
\text { tests improved or stable after } \\
1 \text { year of treatment }\end{array}$ \\
\hline 1 & 53.8 & NE & $3 / 5$ & $1 / 10$ \\
\hline 2 & 77 & NE & $3 / 5$ & $1 / 10$ \\
\hline 3 & 56 & NE & $2 / 3$ & $5 / 10$ \\
\hline 4 & 77 & NE & Treatment stopped & Treatment stopped \\
\hline 5 & 49.3 & NE & Treatment stopped & Treatment stopped \\
\hline 6 & 56.4 & NE & Treatment stopped & Treatment stopped \\
\hline 7 & 92.3 & 8.5 & $3 / 3$ & $6 / 10$ \\
\hline 8 & 88.7 & 9.9 & $4 / 5$ & $3 / 10$ \\
\hline 9 & 93.4 & 13.4 & $4 / 4$ & $6 / 10$ \\
\hline 10 & 93.8 & 0.8 & $2 / 3$ & $2 / 10$ \\
\hline 11 & 87.5 & 5.3 & $2 / 3$ & $1 / 10$ \\
\hline 12 & 78.9 & 11 & $2 / 3$ & $3 / 10$ \\
\hline 13 & 119 & 32.8 & Treatment stopped & Treatment stopped \\
\hline 14 & 95.1 & 25.8 & $3 / 3$ & $9 / 10$ \\
\hline 15 & 83 & 17 & $3 / 2$ & $7 / 10$ \\
\hline 16 & 86.3 & 48.3 & $2 / 2$ & $9 / 10$ \\
\hline 17 & 79.5 & 19.2 & $4 / 4$ & $8 / 10$ \\
\hline 18 & 99.9 & 23.9 & $4 / 3$ & $10 / 10$ \\
\hline 19 & 95.2 & 23.2 & $3 / 4$ & $7 / 10$ \\
\hline 20 & 143.9 & 49.2 & $2 / 2$ & $9 / 10$ \\
\hline $\begin{array}{l}\text { Upper normal } \\
\text { limit of SAI }\end{array}$ & $\begin{array}{l}77.7 \text { (mean plus } \\
2 \mathrm{SD} \text { of control values) }\end{array}$ & & & \\
\hline
\end{tabular}




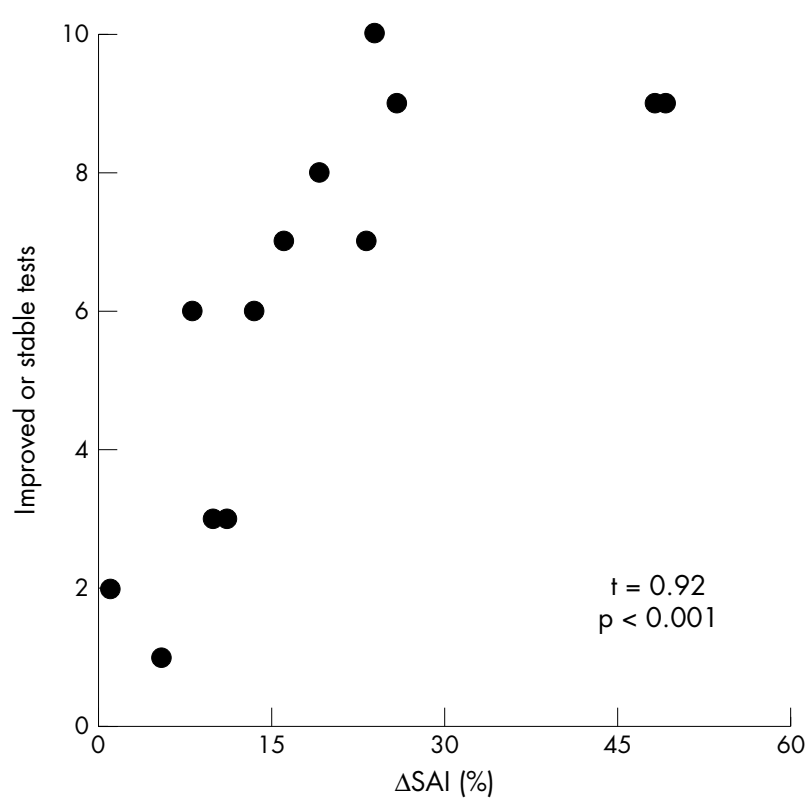

Figure 2 Correlation between the change in SAl (\% of test response) after a single oral dose of rivastigmine and the number of neuropsychological tests $(n)$ improved or stable after 1 year of treatment in individual patients with abnormal SAl. The change in SAl is strongly correlated with the number of tests on which score was improved or unchanged ( $p<0.001$, two tailed Kendall's rank test).

and we cannot exclude the possibility that control subjects age matched with patients may behave differently, even if this seems unlikely because the amount of inhibition produced by afferent stimulation is not influenced by age as demonstrated by the comparable levels of SAI in old and young healthy subjects.

Interestingly, the baseline SAI and the increase in SAI after a single dose of rivastigmine were correlated with the response to long term treatment, as evaluated with the GDS and with an extensive neuropsychological test battery.

Our data suggest that a normal SAI in baseline conditions, or an abnormal SAI in baseline conditions that is not greatly increased by a single oral dose of rivastigmine, is invariably associated with a poor response to long term treatment, while an abnormal SAI in baseline conditions in conjunction with a large increase in SAI after a single dose of rivastigmine is associated with a favourable response to long term treatment in most of the patients.

The amount of change in SAI after a single dose of rivastigmine was also strongly correlated with changes in cognitive measure assessed by the neuropsychological tests after 1 year of treatment. Changes revealed by two tests of long term verbal memory (immediate and delayed recall of RAVLT) correlated with acute changes in SAI produced by rivastigmine. Because these tests are related to long term verbal memory, this correlation is consistent with the hypothesis that the cholinergic systems play a critical role in memory processes and learning. ${ }^{19}$ Indeed, over the last decades, the hypothesis that the cholinergic system is the major neurotransmitter system involved in memory and learning has gained general acceptance. ${ }^{20}{ }^{21}$ We also found a correlation between the acute change in SAI produced by rivastigmine, and the change in phonological verbal fluency after 1 year of treatment. This finding is consistent with the results of a previous study that showed that cholinergic blockade in healthy subjects determines an impairment of verbal fluency. ${ }^{22}$

In agreement with previous studies, none of the clinical and demographic parameters taken into consideration in this study was indicative of the long term response to treatment.
Table 3 Correlations between change in SAI after a single oral dose of rivastigmine and change in performance on each of 10 neuropsychological test (tests based on Kendall's rank correlation)

\begin{tabular}{lcl}
\hline Neuropsychological test & Kendall's $\mathbf{~ t ~}$ & $\mathbf{p}$ \\
\hline MMSE & 0.55 & 0.02 \\
RAVLT, immediate recall & 0.94 & $0.0001^{*}$ \\
RAVLT, delayed recall & 0.73 & $0.004^{*}$ \\
RAVLT, recognition (hits) & 0.2 & 0.54 \\
RAVLT, recognition (false alarms) & -0.46 & 0.11 \\
Immediate visual memory & 0.54 & 0.05 \\
Raven's coloured matrices & 0.22 & 0.41 \\
Constructive praxis & 0.58 & 0.03 \\
Constructive praxis (with landmarks) & 0.28 & 0.32 \\
Phonological verbal fluency & 0.91 & $0.0002^{*}$ \\
\hline
\end{tabular}

*Indicates significance after adjusting for multiple comparisons. Note that all correlations indicate a positive relationship between change in SAI and change in performance (that is, all correlations are positive except the number of false alarm in RAVLT, a reverse measure of performance). MMSE, Mini Mental State Examination; RAVLT, Rey's Auditory Verbal Learning Test.

Our results suggest that the study of SAI and the evaluation of the effects of rivastigmine on SAI could be useful in the management of $\mathrm{AD}$ patients because it is currently impossible to predict an individual therapeutic response in AD patients. Several studies suggest that the evaluation of changes produced by a single dose of tacrine ${ }^{23}$ or by rivastigmine treatment for 1 week $^{24}$ on quantitative EEG could also be useful in predicting the therapeutic efficacy of these drugs. Alhainen and Riekkinen ${ }^{23}$ have shown that a single dose of tacrine produces a more pronounced increase in the absolute alpha power and in the alpha-theta ratio in the subgroup of responders than in the subgroup of non-responders, while Adler et $a l^{24}$ have demonstrated that the subgroup of patients classified as responders had a greater decrease in theta power after 1 week of treatment than the subgroup of non-responders. However, further studies are needed to establish whether the use of the generally available EEG techniques may allow reliable prediction of the likely therapeutic efficacy of rivastigmine in the individual patient. ${ }^{24}$

In conclusion, this neurophysiological study suggests that the evaluation of the non-invasive test SAI in baseline conditions and after a single dose of AChE inhibitors may help in diagnosing a dysfunction of central cholinergic circuits in demented patients and may be useful in identifying those patients who are more likely to respond to long term treatment with AChE inhibitors. However, the study of a larger number of patients is needed before SAI testing can be applied in clinical practice.

\section{ACKNOWLEDGEMENTS}

We thank Maurizio Codispoti for an insightful discussion of statistical methods.

\section{Authors' affiliations}

V Di Lazzaro, A Oliviero, F Pilato, E Saturno, M Dileone, C Marra, F Ranieri, G Gainotti, P Tonali, Institute of Neurology, Università Cattolica, L.go A. Gemelli 8, 00168 Rome, Italy

S Ghirlanda, Department of Psychology, Università di Bologna, Viale Berti-Pichat 5, Bologna, Italy

This work was supported by the Ministero della Sanità (Programma di ricerca finalizzata - Malattia di Alzheimer, 2000 - Regione Lazio Assessorato per le Politiche della Sanità and Programma di ricerca finalizzata - Memoria ed apprendimento motorio dalla fisiopatologia cellulare del danno neurale sperimentale alle nuove tecniche diagnostiche 2003).

Competing interests: none declared 


\section{REFERENCES}

1 Doody RS, Stevens JC, Beck C, et al. Practice parameter: management of dementia (an evidence-based review). Report of the Quality Standards Subcommittee of the American Academy of Neurology. Neurology 2001;56:1154-66.

2 Krall WJ, Sramek JJ, Cutler NR. Cholinesterase inhibitors: a therapeutic strategy for Alzheimer disease. Ann Pharmacother 1999;33:441-50.

3 Di Lazzaro V, Oliviero A, Pilato F, et al. Motor cortex hyperexcitability to transcranial magnetic stimulation in Alzheimer's disease. J Neurol Neurosurg Psychiatry 2004:75:555-9.

4 Di Lazzaro V, Oliviero A, Tonali PA, et al. Non-invasive in vivo assessment of cholinergic cortical circuits in Alzheimer's disease using transcranial magnetic stimulation. Neurology 2002;59:392-7.

5 Di Lazzaro V, Oliviero A, Profice P, et al. Muscarinic receptor blockade has differential effects on the excitability of intracortical circuits in the human motor cortex. Exp Brain Res 2000;135:455-61.

6 Tokimura H, Di Lazzaro V, Tokimura Y, et al. Short latency inhibition of human hand motor cortex by somatosensory input from the hand. J Physiol (Lond) 2000;523(2):503-13

7 Sailer A, Molnar GF, Paradiso G, et al. Short and long latency afferent inhibition in Parkinson's disease. Brain 2003;126:1883-94.

8 Di Lazzaro V, Oliviero A, Pilato F, et al. Normal or enhanced short-latency afferent inhibition in Parkinson's disease? Brain 2004; 127(Pt 4):e8.

9 Ziemann U, Lonnecker S, Steinhoff BJ, et al. Effects of antiepileptics drugs on motor cortex excitability in humans: a transcranial magnetic stimulation study. Ann Neurol 1996;40:367-78

10 Di Lazzaro V, Oliviero A, Meglio M, et al. Direct demonstration of the effect of lorazepam on the excitability of the human motor cortex. Clin Neurophysiol 2000:111:794-9.

11 McKhann G, Drachman D, Folstein M, et al. Clinical diagnosis of Alzheimer's disease: report of the NINCDS-ADRDA Work Group under the auspices of Department of Health and Human Services Task Force on Alzheimer's

Disease. Neurology 1984;34:939-44.
12 Oldfield RC. The assessment and analysis of handedness: the Edinburgh inventory. Neuropsychologia 1971;9:97-113.

13 Kennedy JS, Polinsky RJ, Johnson B, et al. Preferential cerebrospinal fluid acetylcholinesterase inhibition by rivastigmine in humans. J Clin Psychopharmacol 1999;19:513-21.

14 Rey $\mathbf{A}$. Mèmorisation d'une sèrie de 15 mots en 5 rèpètitions. In: Rey $A$, ed. L'examen clinique en psychologie. Paris: Presses Universitaires de France, 1958.

15 Raven JC. Progressive matrices (1947), Sets A, AB, B: Board and Book Forms. London: Lewis, 1949.

16 Villa G, Gainotti G, De Bonis C, et al. Double dissociation between temporal and spatial pattern processing in patients with frontal and parietal damage. Cortex 1990;26:399-407.

17 Reisberg B, Ferris SH, de Leon MJ, et al. Global Deterioration Scale (GDS). Psychopharmacol Bull 1988;24:661-3.

18 Siegel S, Castellan NJ Jr. Nonparametric statistics for the behavioral sciences, 2nd ed. New York: MacGraw-Hill, 1988.

19 Francis PT, Palmer AM, Snape M, et al. The cholinergic hypothesis of Alzheimer's disease: a review of progress. I Neurol Neurosurg Psychiatry 1999;66:137-47.

20 Bartus RT. Evidence for a direct cholinergic involvement in the scopolamineinduced amnesia in monkeys: effects of concurrent administration of physostigmine and methylphenidate with scopolamine. Pharmacol Biochem Behav 1978;9:833-6.

21 Everitt BJ, Robbins TW. Central cholinergic systems and cognition. Annu Rev Psychol 1997:48:649-84.

22 Aarsland D, Larsen JP, Reinvang I, et al. Effects of cholinergic blockade on language in healthy young women. Implications for the cholinergic hypothesis in dementia of the Alzheimer type. Brain 1994;117:1377-84.

23 Alhainen K, Riekkinen PJ Sr. Discrimination of Alzheimer patients responding to cholinesterase inhibitor therapy. Acta Neurol Scand Suppl 1993;149:16-21.

24 Adler G, Brassen S, Chwalek K, et al. Prediction of treatment response to rivastigmine in Alzheimer's dementia. I Neurol Neurosurg Psychiatry 2004;75:292-4. 